Revue de l'Institut des langues et cultures

d'Europe, Amérique, Afrique, Asie et Australie

$13 \mid 2010$

Les voies incertaines de la démocratisation

\title{
La Transition espagnole : grands récits et état de la question historiographique
}

The Spanish Transition (1975-1982): A Political Myth Revisited

\section{Christian Demange}

\section{OpenEdition}

Journals

Édition électronique

URL : https://journals.openedition.org/ilcea/874

DOI : 10.4000/ilcea.874

ISSN : 2101-0609

\section{Éditeur}

UGA Éditions/Université Grenoble Alpes

\section{Édition imprimée}

ISBN : 978-2-84310-181-6

ISSN : $1639-6073$

Référence électronique

Christian Demange, « La Transition espagnole : grands récits et état de la question

historiographique », ILCEA [En ligne], 13 | 2010, mis en ligne le 30 novembre 2010, consulté le 21

septembre 2021. URL : http://journals.openedition.org/ilcea/874 ; DOI : https://doi.org/10.4000/ilcea 874

Ce document a été généré automatiquement le 21 septembre 2021.

(c) ILCEA 


\title{
La Transition espagnole : grands récits et état de la question historiographique
}

\author{
The Spanish Transition (1975-1982): A Political Myth Revisited
}

\author{
Christian Demange
}

1 Un colloque consacré aux voies incertaines de la démocratisation, et regardant plus particulièrement les phénomènes de transition et de consolidation de la démocratie en Amérique latine, ne pouvait guère faire l'économie d'une référence à l'Espagne, la première à avoir conduit ce processus nouveau, car non révolutionnaire, de passage d'une dictature à une démocratie. D'autant plus que cette Transition espagnole est vite devenue non seulement un modèle pour de nombreux pays d'Amérique latine et d'Europe de l'Est confrontés à cette même situation de passage, mais aussi le nouveau mythe sur lequel repose la jeune démocratie espagnole. Un mythe fortement remis en question aujourd'hui, non seulement par l'importante production historiographique espagnole et française sur laquelle je m'appuie largement - n'étant pas moi-même un spécialiste de cette question - mais aussi par la réalité du pays elle-même.

\section{L'historiographie initiale}

\section{Quelques repères}

2 On observe une prolifération des travaux sur la Transition espagnole, ce qui peut s'expliquer par l'intérêt personnel des historiens pour cette période qu'ils ont vécue pour beaucoup d'entre eux, mais aussi par les encouragements à travailler sur ce sujet suite au succès politique inattendu du projet réformiste et à la consolidation indiscutable du nouveau régime démocratique en Espagne. La Transition est une success story qui appelle des commentaires. 
3 Les premiers ouvrages développent une conception personnaliste et volontariste, qui met en avant de grandes figures, les protagonistes de la Transition, et qui réduit le processus à une série de pactes entre les élites issues de l'ancien régime et de l'opposition démocratique, dont l'objectif commun est de dérouler au mieux la feuille de route qui conduira le pays vers la démocratie. Du coup, la société est réduite au rang de spectatrice. En 1978, Juan Cortes Cabanillas, dans Crónica de Juan Carlos Rey. De la Restauración a la Constitución (Alce, 1978), met l'accent sur le roi comme protagoniste de la Transition dont il offre une vision optimiste, la décrivant comme un fait extraordinaire et une exception dans l'histoire sans doute parce que, avec la Transition, il a l'impression que l'Espagne a liquidé définitivement la Guerre civile. En 1979, Raymond Carr et Juan Pablo Fusi, dans España, de la dictadura a la democracia (Barcelone, Planeta, 1979), profitent de la Transition pour réactualiser les thèses libérales: la Transition prend ses racines dans les transformations de la société espagnole sous le franquisme, surtout pendant les années 1960 et 1970. La Transition est la conséquence logique et inévitable de la modernisation de la société qui finit par conduire à la modernisation des formes politiques. L'idée est que toute dictature devient un jour obsolète et finit par être balayée par les forces de la démocratie et du marché. En 1984, Rodolfo Martin Villa, figure politique de la Transition, publie Al servicio del Estado (Barcelone, Planeta, 1984) et soutient l'idée que ce sont les réformistes du franquisme qui ont exécuté le projet politique du roi et qui ont contribué à la naissance d'une démocratie pour tous les Espagnols, en restant dans le cadre légal de la dictature. En 1986, Paul Preston, dans El triunfo de la democracia en España (1969-1982) (Barcelone, Plaza y Janés, 1986), tente de rétablir un certain équilibre entre le rôle joué par l'ensemble de la société et celui des élites, dont l'historiographie dominante magnifiait déjà le rôle dans le processus démocratique. Mais la vision dominante s'impose encore dans les ouvrages de Vicente. Palacio Atard, Juan Carlos I y el advenimiento de la democracia en España(Madrid, Espasa-Calpe, 1989) - qui defend la thèse que le changement est l'œuvre de trois protagonistes : le roi qui marque le cap, Adolfo Suárez qui est au gouvernail et Torcuato Fernández Miranda qui sonde les écueils pour les éviter - et de Ch. T. Powell, El piloto del cambio. El rey, la monarquía y la Transición a la democracia (Barcelone, Planeta, 1991), qui présente encore le roi comme le moteur du changement, comme celui qui a été capable d'éliminer les résistances franquistes et de contenir les exigences des partisans de la rupture.

4 Il faut attendre la publication, dans la première moitié des années 1990,des ouvrages de Javier Tusell (La Transción española a la democracia, Madrid, Historia 16, 1991 ; Historia de la Transición, 1975-1986, Madrid, Alianza Ed., 1996) et de Manuel Redero San Román(Transicion a la democracia y poder político en la España postfranquista (1975-1978), Salamanca, 1993 ; La Transición a la democracia en España, Madrid, Marcial Pons, 1994), pour voir s'ouvrir le débat scientifique sur la Transition. Si Manuel Redero San Román accepte que le processus soit essentiellement politique, il refuse d'un point de vue méthodologique l'autonomie des forces politiques et institutionnelles, et introduit d'autres variables, comme l'économie, la société et la culture.

\section{Deux grands récits qui hébergent chacun plusieurs versions}

5 L'histoire de la Transition donne lieu à deux grands récits qui tendent à la simplifier à l'excès et à la réduire souvent à une histoire factuelle - au détriment de modèles explicatifs plus complexes - dans laquelle prédominent les points de vue narratifs et 
unidirectionnels. La raison en est que pour que l'histoire de la Transition soit assimilable et puisse remplir sa fonction de catéchisme laïc de la nouvelle démocratie, il a fallu donner la priorité aux interprétations simples. Ces deux grands récits, avec plusieurs versions chacun, sont en lutte pour l'hégémonie, et donne l'impression d'un débat et d'une pluralité.

6 Le premier de ces récits, le modèle libéral classique, met en jeu des forces souterraines puissantes qui poussent historiquement les sociétés vers la démocratie. Il peut se définir ainsi : le changement politique est associé à la modernisation sociale et économique des années 1960-1970 ; la Transition est la conséquence d'une modernisation de la société (modernisation impulsée par la dictature dans ses dernières années); une société qui finit par demander la modernisation des formes politiques: la volonté des citoyens, la mobilisation des masses, les accords politiques, dans ce schéma, sont subordonnés à la grande vague démocratique qui a son épicentre dans la libéralisation économique et l'ouverture à l'extérieur. Ce récit se décline en trois versions. La version des marxistes orthodoxes pour lesquels la Transition a joué le rôle que n'a pas pu jouer la révolution bourgeoise en Espagne, faute d'avoir eu lieu à cause des guerres civiles. La version des libéraux pour lesquels la tendance naturelle des dictatures est d'être balayées par les forces puissantes de la démocratie et du marché. La version plus conservatrice du libéralisme, selon laquelle la dictature a rempli la mission historique que le libéralisme politique espagnol du xIx siècle et le radicalisme du $\mathrm{xx}^{\mathrm{e}}$ siècle avaient été incapables de remplir: la modernisation de l'Espagne qui a mis fin aux extrémismes et aux guerres civiles, et qui a assis les bases du vivre ensemble. La dictature est alors considérée comme un moindre mal qui a permis la modernisation du pays et l'évolution des citoyens vers la modération, créant ainsi un large espace politique pour le centre. De plus la dictature aurait permis à l'Espagne d'intégrer l'Europe politique et économique.

7 Le second récit met au centre du processus de transition la volonté politique. Selon ce récit, la transition dépend de l'action consciente des individus, des groupes politiques, des groupes sociaux et non de forces souterraines. La dictature n'était pas condamnée d'avance à disparaitre après la mort de Franco et le changement de régime dépendait d'une opposition forte, d'un engagement clair en faveur de la démocratie, et de la prise des bonnes décisions à chaque moment du processus. Ce récit se décline en deux versions. Une version plutôt conservatrice qui accorde un poids énorme dans le processus à des individus concrets qui sont le véritable moteur de la Transition - mythification du rôle du roi, de Adolfo Suarez, de Santiago Carrillo, de Felipe González, etc. - ainsi qu'une importance extrême à l'action de ces individus qui interviennent dans des négociations secrètes et passent des accords secrets. Dans ce schéma, on néglige tout ce qui, habituellement, explique les changements: pas d'analyse en profondeur des organisations politiques, des mouvements sociaux, des conditions économiques, des influences extérieures, des changements dans l'éducation, la culture ou la religiosité. L'autre version, que certains qualifient de marxiste populiste, et qui est portée aussi par les nationalismes périphériques, accorde le rôle principal aux masses qui, représentées par des organisations politiques et syndicales de l'opposition parfois, se manifestent directement dans les grèves et lors des grandes vagues de protestation. Ces masses sont mises en avant aussi pour leur modération, leur sagesse, leur maturité. Dans les autonomies, on donne ce premier rôle aux peuples qui luttent pour défendre leurs droits historiques et leur droit à l'autonomie. Dans cette version ce sont les aspirations de la société civile qui produisent la lutte pour la démocratie. Toutes ces versions ont leur 
place dans l'historiographie officielle dans la mesure où elles ne remettent pas en cause le modèle analytique de la Transition, le passage par la loi et par la cogestion : l'essentiel est préservé.

8 À partir de 1997, la nouvelle historiographie commence un travail de révision de la Transition (thèses, travaux, séminaires, congrès, journées d'études prennent comme objet la Transition) avec l'intention de rétablir la vérité car, entre-temps, cette première production historiographique a donné naissance à un véritable mythe.

\section{La Transition : le nouveau mythe politique au fondement de l'identité nationale et démocratique de l'Espagne d'aujourd'hui}

\section{Pourquoi y a-t-il eu idéalisation des résultats de la Transition et mythification de celle-ci ?}

9 La période est vécue comme une période de changements profonds et rapides. Il y a une accélération de l'histoire qui place la dictature dans un passé qui semble déjà lointain et qui oblige à construire un nouvel imaginaire du pouvoir et de la politique. Il y a de plus à cette époque-là dans la société espagnole une forte volonté de se concentrer sur la démocratie et d'éviter les affrontements passés, ce qui conduit au choix du consensus politique. Les modalités de la Transition font de celle-ci le grand succès collectif des Espagnols. Le dernier en date, trop lointain déjà pour être opérationnel en termes de mémoire collective, avait été la Guerre d'indépendance contre Napoléon, en 1808-1814. La réconciliation qu'opère la Transition peut devenir le nouveau grand récit qui remplacera celui qui s'était imposé jusqu'alors à tous : celui de la Guerre civile, tuerie fratricide et jugée désormais inutile. La Transition devient ainsi un moment pour la mémoire, un moment dont les protagonistes doivent être célébrés, immortalisés en tant qu'architectes du nouveau temple constitutionnel issu du consensus politique. Une constitution que l'on célèbre en grande pompe, en 2003 à l'occasion du $25^{\mathrm{e}}$ anniversaire, dans les journaux. $A B C$ , El Mundo, El País en distribuent des exemplaires et multiplient les numéros spéciaux, avec les témoignages des pères de la constitution, qui se félicitent que la Transition ait tout changé sans rien casser : un véritable miracle. Dans ce contexte, la monarchie, fruit de la Transition, est la grande gagnante. Elle est associée à la stabilité politique, à la prospérité économique et au progrès avec l'incorporation de l'Espagne dans l'ensemble politique et économique européen, qui était un des objectifs de la Transition du point de vue des héritiers de l'ancien régime. Cette nouvelle équation, « monarchie = démocratie ", montre les profonds changements qui s'opèrent dans la mémoire nationale à l'occasion de la Transition. Il y a bien un avant et un après la Transition.

\section{Mythe, historiographie et pouvoir}

10 Le mythe de la Transition est lié au pouvoir politique, qui voit en la mémoire collective un puissant instrument pour configurer l'avenir et qui a été présent dans l'historiographie par le biais du financement préférentiel, de la reconnaissance publique, de sa politique commémorative et des manuels scolaires. Ce rapport de l'historiographie au pouvoir conditionne la diffusion du discours historiographique dominant qui frôle parfois 
l'endoctrinement, comme lors du $30^{\mathrm{e}}$ anniversaire de la restauration de la monarchie en 2005, alors que le $75^{\mathrm{e}}$ anniversaire de la proclamation de la République, première véritable expérience démocratique, un an plus tard, passe presque inaperçu, et alors que le discours historiographique critique peine à se faire entendre dès lors qu'il aborde des sujets tabous comme le coup d'État militaire du 23 février 1981 et ses implications, ou les liens de la famille royale avec Franco. Le pouvoir politique, puissamment relayé par le pouvoir médiatique, a célébré les dates clefs. En 1986 par exemple, le PSOE de Felipe Gonzalez fait certes la promotion de la mémoire réconciliée à l'occasion du $50^{\mathrm{e}}$ anniversaire de la Guerre civile, mais il assure un service minimum alors que pour les 10 ans de l'accession au trône de Juan Carlos, il avait sorti le grand jeu. Globalement, la Transition donne lieu à un discours officiel, dominant et omniprésent, à une mémoire sociale construite par les élites avec des objectifs précis: se légitimer au pouvoir et légitimer un certain pouvoir.

\section{Un mythe politique d'une transition exemplaire au service d'une nouvelle identité démocratique}

Voyons d'abord les éléments du mythe. La Transition espagnole est idéale et exemplaire parce qu'elle est une transition négociée, graduelle et pacifique, qui repose sur des élites responsables, modérées et bienveillantes, engagées dans la démocratie. Elle se fonde sur un consensus politique qui a permis, entre autres choses, de sceller un pacte d'oubli pour faire table rase du passé (loi d'amnistie) et regarder vers un avenir démocratique. Elle repose donc sur la réconciliation nationale, élément nécessaire à la conciliation politique, à la cogestion.

12 Cette représentation de la transition devient très vite le mythe de la nouvelle Espagne et le fondement de son identité démocratique parce que le pouvoir a voulu asseoir sur des bases solides la nouvelle identité démocratique et pro-européenne que devaient faire leur les nouvelles générations d'Espagnols. Avec le mythe de la Transition, les nouvelles valeurs de la démocratie espagnole deviennent la clairvoyance des dirigeants, la maturité et la modération du peuple, le rejet des idéologies trop marquées (extrémistes), le pragmatisme politique, la réconciliation nationale, l'oubli, le pardon, le culte de la stabilité politique et l'horreur des conflits - même idéologiques-, l'équation monarchie = démocratie et la perception du roi comme symbole fédérateur de la nation. Mais l'historiographie universitaire à elle seule aurait été incapable d'élever au rang de mythe national la Transition. C'est le relais de l'historiographie médiatique qui a donné cette dimension et cette puissance à la mémoire sociale de la Transition.

\section{Le rôle de l'historiographie médiatique}

13 La puissance de la mémoire sociale de la Transition ne s'explique pas que par les manuels scolaires. Elle s'explique surtout par la prégnance des récits que véhiculent les médias (presse et télévisions) qui ont une forte capacité à simplifier les images du passé et à les inscrire dans le souvenir de larges secteurs de la collectivité. L'histoire comme reconstruction du passé n'est plus seulement l'affaire des historiens professionnels, même si les résultats de leurs travaux débouchent sur les manuels scolaires. Les journalistes s'en chargent aussi, et depuis quelques années les échanges sont nombreux entre les deux milieux professionnels : Victoria Prego, journaliste, écrit une histoire de la 
Transition tandis que les historiens, tel Santos Julia, collaborent régulièrement dans la presse. Il y a donc deux historiographies, l'universitaire et la médiatique.

Cette historiographie médiatique est d'autant plus puissante qu'elle reprend le discours tenu par l'école, comme c'est le cas pour la Transition espagnole où les deux discours coïncident presque toujours et se renforcent, rendant très difficile toute modification $\mathrm{du}$ discours officiel par le débat intellectuel et la recherche universitaire récente. Voyons quelques exemples. En 1985, la RTVE diffuse le documentaire Operación Triunfo qui contribue à socialiser fortement la version officielle de la Transition : le roi Juan Carlos avait l'idée très claire que l'Espagne devait se transformer en démocratie quand il a accédé au trône et il y avait déjà travaillé, tant à l'intérieur qu'à l'étranger. La série télévisée de Victoria Prego, intitulée La Transición (los años más decisivos de nuestra historia reciente), 1993, en treize chapitres - diffusée par la suite sous forme de cassettes VHS avec l'aide du ministère de la Culture, puis par le journal El Pais, puis rediffusée gratuitement à partir de novembre 2003 par l'hebdomadaire Tiempo, sous forme de DVD - est un autre exemple de la puissance des médias. On peut noter qu'il sert à expliquer la période dans les collèges, sans autre apport critique. Cette série, emblématique du genre, produite par la télévision publique et diffusée sur la seconde chaîne entre juillet et octobre 1995, a demandé une préparation de plus de 4 ans. Le documentaire, dont le projet est né en 1988, est terminé en 1992/1993, mais il est gelé par la direction avant d'être finalement diffusé peu avant les élections. Cette série relève d'une conception historique et historiographique événementielle et positiviste : elle tend à expliquer l'histoire comme une série d'événements ponctuels et comme une succession d'actions concrètes de personnages individuels et héroïques qui sont membres des hautes sphères du pouvoir (roi, gouvernants, généraux) ou qui appartiennent aux élites de la société de l'époque, et elle propose une histoire tournée vers le progrès continu. Elle reprend la thèse canonique de la Transition. Le processus de la transition nous apparaît comme un concert, avec un compositeur, le roi Juan Carlos, qui aurait écrit la partition à l'époque où il vivait à l'ombre du général Franco, un chef d'orchestre, Adolfo Suarez, un orchestre symphonique : la classe politique, un premier violon, Santiago Carrillo et une première flûte: Felipe Gonzalez, un concert avec un public attentif et tout acquis: le peuple espagnol, même si un ou deux perturbateurs, incapables d'apprécier la beauté de la musique ou impatients, peuvent venir troubler l'écoute du reste du public. La transition apparait comme un processus exclusivement dirigé d'en haut et par ceux d'en haut, qu'il s'agisse de l'appareil d'État ou des partis politiques importants et encore dans la (semi) clandestinité. On prête des intentions démocratiques au roi avant la mort de Franco ; on affirme à plusieurs reprises que le roi savait ce qu'il voulait, comment il allait procéder et avec qui. On ne fait référence qu'aux dirigeants des grands partis. On n'évoque jamais les peurs, les incertitudes ni les différentes initiatives qui n'ont jamais vu le jour mais qui étaient là. On surdimensionne le rôle joué par Felipe González et le PSOE, le syndicat socialiste UGT (mais pas par le syndicat, alors d'obédience communiste, Commissions ouvrières). Cette dimension philo-socialiste de la série, élaborée sous les gouvernements socialistes et avec Pilar Miró à la tête de Radio Télévision espagnole montre clairement le lien de l'historiographie médiatique avec le pouvoir politique. Tout le documentaire est un chant de louange au processus de transition, sans la moindre note critique. En 1995, à l'occasion du $20^{\mathrm{e}}$ anniversaire de la Transition, le journal El Pais diffuse une collection de livrets mêlant témoignages et réflexions de personnalités, d'historiens et de témoins d'exception. L'ensemble sera repris dans un livre en 1996, 
Memoria de la Transición (Taurus). L'objectif déclaré du livre est de faire partager cette mémoire de la Transition aux nouvelles générations. L'idée développée est que la démocratie espagnole n'est ni la plus vertueuse ni la plus vicieuse, que l'Espagne a rejoint le peloton de tête des pays européens, et que cette Transition est un succès. Pour le $30^{\mathrm{e}}$ anniversaire, le même quotidien donne une image encore plus positive de cette Transition, et du roi qui recueille les avis favorables de tous, y compris du républicain Gaspar Llamazares et des communistes français de l'Humanitéqui mesure le chemin parcouru par l'Espagne depuis la fin du franquisme.

Pour imposer ce discours, il a fallu écarter les discours dissidents susceptibles de remettre en question cette vision officielle. Ainsi, en 1981, le documentaire de Cecilia et José Juan Bartolomé, Después de..., devient un documentaire maudit. Production indépendante, réalisée sans grands moyens, le film est déposé à la Direction générale du Cinéma peu avant le coup d'État du 23 février 1981. L'Administration le retient pendant des mois et lui supprime toutes les subventions auxquelles il avait droit, et ses auteurs sont même inquiétés par la justice et accusés de délit au motif que, d'une certaine façon, ils avaient pronostiqué le coup d'État de Tejero. Le film (tourné en 1979-1980) posait le problème d'un processus entrepris sans rupture qui laissait de l'espace pour une renaissance militante du franquisme et pour l'expression d'hommages nostalgiques à Franco. Il évoquait aussi l'usure prématurée des partis politiques, l'escalade des violences terroristes, sans oublier une interrogation sur les Forces armées - on est à quelques semaines du 23 février 1981. Il faisait aussi allusion au désenchantement, ou plutôt au mécontentement des secteurs progressistes face aux changements insuffisants. Il donnait la parole aux gens de la rue et faisait une grande place aux protestations, aux meetings, de gauche, d'extrême gauche, d'extrême droite. Il limitait au maximum les commentaires omniscients, préférant les témoignages des mécontents. On était loin de l'image canonique de la Transition. Plus récemment, ce sont les documentaires sur la nature passée du régime franquiste avec lequel a pactisé la Transition qui se confronte à la résistance des médias. Le documentaire Les enfants perdus du franquisme (2002) de la journaliste catalane Montsé Armengou (conseil scientifique de l'historien Ricard Vinyes), n'avait toujours pas été diffusé par RTVE en avril 2004. Seules les télévisions catalane et basque l'avaient diffusé à une heure de grande écoute (l'Andalousie l'ayant diffusé à une heure de faible écoute).

Rien d'étonnant dans ces censures, car ce qui est en jeu aussi dans cette version officielle de la Transition, c'est la reconfiguration de la mémoire historique. La Transition est un changement politique qui a conduit à la démocratie mais aussi un laboratoire de révision historiographique du passé d'où a surgi une version officielle de l'histoire de l'Espagne au $\mathrm{xx}^{\mathrm{e}}$ siècle, sous les auspices de ceux qui avaient commandé le bateau de la Transition. Cette vision partisane de la Guerre civile et du franquisme est basée sur le principe de l'équidistance, selon lequel tout le monde avait sa part de responsabilité dans le déclenchement du conflit. Le régime qui en était issu, bien que condamnable, devenait une sorte d'expiation des fautes collectives. Cette reconfiguration du passé est une nécessité politique sous la Transition. En effet, la seule façon pour les auteurs de la Transition de surmonter la division entre Espagnols était d'admettre que chacun avait une responsabilité et que le mieux était d'oublier le passé pour regarder l'avenir. Ce qui fait de l'Espagne, selon le journaliste Gregorio Morán, auteur de El precio de la Transicion (Barcelone, Planeta, 1991), un royaume dont le peuple a perdu sa mémoire. 
17 Cet état de fait explique la résurgence d'une mémoire condamnée jusqu'ici au silence, et qui a soif de se raconter pour contrecarrer cette vision partisane de l'histoire, imposée par les modalités de la transition à la démocratie et diffusée abondamment par les médias. C'est l'histoire de Remedios Montero qui, n'en pouvant plus de voir l'ignorance de la jeunesse espagnole sur sa propre histoire, décide à 78 ans de témoigner de son expérience de maquisarde dans un récit repris par Alfons Cervera, Historia de Celia. Memorias de una guerrillera antifascista (2004).

18 Malgré tout, les médias imposent très clairement la version officielle de la Transition. Une enquête de 2000 montre que la version officielle de la Transition diffusée par les médias a réussi à s'imposer dans la mémoire sociale en fixant des clichés et des stéréotypes interprétatifs qui résistent très bien aux nouveaux apports de l'historiographie. La Transition est toujours motif de fierté, le roi a toujours le premier rôle, aux côtés de A. Suarez, et on continue à personnaliser le processus au détriment des acteurs collectifs, par exemple. Quant au franquisme comme étape historique, les Espagnols en disent qu'il y a eu de bonnes et de mauvaises choses. Sans doute est-ce dû aux efforts de l'historiographie médiatique (mais pas seulement) pour faire démarrer la transition durant l'étape tardo-franquiste, étape décisive pour la réflexion de certains personnages clef de l'ancien régime qui auraient alors pris la décision de contribuer à la naissance d'un régime pluraliste et non totalitaire. Franco n'étant plus que le dernier obstacle à leur bonne volonté.

\section{De la transition-modèle au modèle de transition}

La Transition espagnole va se constituer progressivement en un nouveau modèle, en une nouvelle façon de concevoir les sorties des dictatures, une sorte de troisième voie qui va séduire l'étranger, de l'Amérique latine aux pays de l'est de l'Europe. L'Espagne offre très tôt au monde son modèle de transition, idéalisée dans le discours officiel. Pour elle c'est d'abord un élément de reconnaissance internationale: la réussite de son passage à la démocratie lui offrait une revanche sur l'histoire en effaçant des siècles de "légende noire ", une légende dont le dernier épisode avait été la Guerre civile, qui renvoyait à son incapacité à résoudre pacifiquement les conflits et à articuler une démocratie moderne. $\mathrm{Au}$ passage, cette reconnaissance internationale lui permettait de renforcer le mythe sur le plan intérieur. La bonne fortune de l'expérience historique de la Transition espagnole est d'avoir eu lieu à un moment ou le modèle analytique de la révolution, qui jusqu'à la fin des années 1970 avait servi à presque tout (révolutions nationale, coloniale, industrielle et même démographique) mais aussi à envisager les sorties des régimes totalitaires, arrivait à épuisement. Les dernières décennies $\mathrm{du} \mathrm{xx}^{\mathrm{e}}$ siècle sont marquées par de profonds changements. Elles voient la fin de la logique de la guerre froide, la fin de la poussée socialiste, la fin de l'utopie révolutionnaire et même la fin du paradigme du progrès. Dans ce contexte, le concept de révolution qui, depuis la Révolution française, prétendait expliquer tous les processus de changement au sein d'une société (prise du pouvoir politique par la force, en invoquant des idéaux progressistes, et avec l'appui majoritaire du peuple) entre en crise. Le passage de la dictature à la démocratie, en Espagne, apporte un nouveau modèle de compréhension des changements politiques : le modèle de la transition, qui jusqu'ici avait servi a expliqué des changements longs et en profondeur (système féodal par exemple). La transition est graduelle, sans rupture révolutionnaire - elle utilise les anciennes institutions -, basée sur le dialogue et le 
consensus, ce qui exclut la violence et l'instabilité politique tout en donnant des résultats relativement rapides et satisfaisants. Elle se fonde sur une politique de réconciliation nationale et procède par réformes et non par rupture. Négociée entre élites responsables, elle évite à la fois les coups d'État réactionnaires et les mobilisations populaires sources de déstabilisation. Et enfin elle laisse intactes les bases du pouvoir social et économique et n'affecte que les institutions et les formes politiques. Ce modèle permet donc de s'éloigner à la fois de la dictature et de la révolution, devenues toutes deux indésirables au temps de la post-modernité. L'issue de la dictature n'est plus forcément la révolution : il y a une troisième voie, une alternative à la révolution qui, de plus, rencontre l'assentiment des peuples, désireux de modernisation et d'Europe (deux réalités liées à l'idée de démocratie). L'apport de la Transition espagnole est donc d'avoir permis de théoriser un modèle alternatif, pacifiste, rapide et réformiste, d'accès à la démocratie à partir de dictatures et de régimes autoritaires, dans une conjoncture d'émergence du capitalisme global. Et ce, bien qu'elle se caractérise par une grande pauvreté en idées, un pragmatisme technocratique, la disparition des mouvements sociaux et des utopies politiques, l'apathie et le désenchantement des citoyens. Dans les années 1990, la Tchécoslovaquie, la Roumanie, la Pologne, la Bulgarie et la Russie se référeront au modèle espagnol, comme l'avaient fait avant eux l'Argentine, le Chili, l'Uruguay, puis les pays d'Amérique centrale.

\section{La révision historiographique de la Transition du début du $X X I^{e}$ siècle}

La nouvelle historiographie qui naît vers 1997, date à laquelle les historiens du temps présent lancent en Espagne les premières thèses, les premiers congrès et journées d'études consacrés à la Transition, s'attache à corriger la vision mythique et officielle de la Transition espagnole véhiculée par les médias et les institutions. Elle sera bientôt portée par le mouvement social qui naît autour de la récupération de la mémoire historique, ce qui lui donnera une plus grande visibilité. En s'attaquant au mythe de la Transition, elle remet en cause du même coup le mythe fondateur de la démocratie espagnole, ce qui explique largement les résistances auxquelles elle se confronte dans les médias, et nous en avons vu quelques exemples. Il y a, sous-jacent à cette révision historiographique de la Transition, très liée à la problématique de la mémoire historique occultée sous le franquisme et les 20 premières années de la Transition, la volonté de refonder la démocratie espagnole sur d'autres paramètres, sur d'autres valeurs, enracinées davantage dans le passé de la seconde République, la grande absente de la Transition.

\section{Les raisons d'un retour sur l'historiographie de la Transition}

21 Ce qui amène les nouveaux historiens à se pencher sur l'histoire de la Transition, outre le fait du développement d'une histoire du temps présent, c'est d'une part le constat de l'existence d'une pluralité de mémoires reléguées qui émergent; c'est d'autre part la critique croissante du discours historique et historiographique dominant, et la perception généralisée que l'Espagne est face à la fin d'un processus de transition qui n'arrive pas à se consolider définitivement (problèmes des nationalismes périphériques et de la mémoire de la Guerre civile). Les modèles d'explication historiques (l'idéalisation de la 
transition, l'idée d'un passé où les fautes s'équilibrent) sont de moins en moins crédibles et sont remis en question : l'incorporation à l'historiographie de la notion des droits de l'homme fait ressortir les anomalies qui ont accompagné l'historiographie espagnole contemporaine, une fois qu'est documentée la nature répressive de la dictature franquiste grâce aux travaux que conduisent de nombreux jeunes historiens espagnols à partir des archives qui commencent à s'ouvrir. On fait ici allusion aux travaux sur les camps de concentration, les bataillons disciplinaires de travailleurs, les enfants de la Guerre, les brigades internationales, les résistants antifascistes des maquis, les prisons de femmes, etc., c'est-à-dire à des catégories de victimes oubliées jusqu'ici. Cette historiographie récente montre que la dictature franquiste n'a pas été ce qu'en dit la révision historique élaborée au temps de la Transition; ce qui pose le problème d'une transition qui fonde la démocratie sur l'oubli et l'amnistie de crimes de guerre, voire même de crimes contre l'humanité, et sur la négation des droits de l'homme. La reconstruction de la mémoire du franquisme suffit à elle seule à démythifier, ou pour le moins interroger, la Transition.

\section{Les grandes figures}

22 Elles sont fort nombreuses. Cependant, les grands agitateurs ou animateurs de l'historiographie sur la Transition, ceux qui organisent et provoquent le débat, sont d'une part les historiens et d'autre part les politologues. Parmi les historiens, on peut citer Carme Molinero, La Transición, treinta años después (Barcelone, Península, 2006), dont la préoccupation est de réduire la distance qui existe entre les connaissances universitaires et les connaissances socialisées. Elle se bat pour que les historiens contrecarrent le bruit médiatique et les lectures intéressées de la Transition. Mais aussi Abdon Mateos, La España del presente: de la dictadura a la democracia (Madrid, Asociación de historiadores del presente, 2006) et Rafael Quirosa-Cheyrouze y Muñoz, éditeur de la Historia de la Transición en España. Los inicios del proceso democratizador (Biblioteca Nueva, Madrid, 2007). Quirosa Cheyrouze a organisé un premier congrès en 2000 sur le thème de la Transition, un second en 2005 (d'où est tiré l'ouvrage cité) qui a permis de mettre l'accent sur la mobilisation des acteurs sociaux, sur le rôle du peuple dans le processus démocratique, un troisième en 2007 consacré au rôle des médias. Le quatrième, qui était prévu pour novembre 2009, devait, selon l'appel à communication, s'attacher à

corriger une historiographie qui donne trop d'importance aux décisions adoptées par les dirigeants politiques, particulièrement ceux qui étaient issus de la dictature précédente, et qui présente la transition comme un plan élaboré par les élites. Ce congrès, intitulé Société et mouvements sociaux, essaiera de mettre en avant le rôle des acteurs sociaux qui ont conditionné par leur action et leur pression les décisions des élites.

Comme on le voit, la perspective n'est pas révolutionnaire, mais elle s'attache à rétablir une certaine vérité qui dérange parce qu'elle remet partiellement en cause la vision officielle. Un travail comme celui que fait Gustavo Muñoz à propos du discours du PSOE sur la réconciliation («La problemática del pasado y el discurso sobre reconciliación nacional del socialismo español durante el franquismo y la primera transición: su relacion con la acción política del partido", Hispania Nova, $\mathrm{n}^{\circ}$ 6, dossier, 2006) permet de montrer que le parti a eu successivement trois discours différents sur le problème de la réconciliation, en fonction de ses choix stratégiques, et qu'à un moment donné, pour être présent en bonne place sur l'échiquier politique, le parti a renoncé à ses convictions et a décidé de défendre le 
processus de transition imposé par Suárez parce qu'il en bénéficiait pleinement, ce qui l'a conduit à sacrifier l'héritage républicain dont il était porteur et par là même à redessiner en profondeur la dialectique de la confrontation : l'Espagne rouge et l'Espagne bleu font la paix pour refonder l'État sur le consensus et s'affronter ensemble à la nouvelle antiEspagne extrémiste (de droite ou de gauche) et aux nationalismes indépendantistes.

Plus globalement, cette historiographie combat les tentatives de récupération des faits historiques au bénéfice d'intérêts politiques présents. Ils combattent tout spécialement une interprétation de l'histoire, celle qui décrit la Transition comme un processus exemplaire, exportable et parfaitement organisé, qui aurait suivi une feuille de route tenue par le roi : la vision officielle. Ils s'attachent à montrer : $1^{\circ}$ que la transition n'aurait pas eu le même destin sans la pression sociale et populaire qui a aidé à réduire la résistance des ultras du franquisme et à surmonter les menaces terroristes (extrême droite, ETA, Grapo), et qui a fait basculer vers la démocratie les secteurs sociaux et les hommes politiques recyclés du franquisme (ce qui s'est traduit par la légalisation du PCE et les accords de la Moncloa par exemple); $2^{\circ}$ que le processus a été caractérisé au contraire par les improvisations, les incertitudes plutôt que par la planification ; $3^{\circ}$ qu'il existait pour l'Espagne post-franquiste au moins cinq projets (de continuité, de pseudoréforme, de réforme, de rupture, et enfin de révolution) qui ont eu leur influence sur la dynamique du processus de démocratisation ; $4^{\circ}$ que de multiples obstacles s'opposaient à la consolidation de la démocratie : les secteurs les plus intransigeants du franquisme, les violences terroristes, la crise économique (chômage et conflits sociaux), les demandes d'autonomie, la faiblesse des partis politiques, la culture politique défaillante des Espagnols, les souvenirs de la Guerre civile, etc. Tous ces problèmes ont produit des conflits et de la violence : les récents travaux de Sophie Baby montrent qu'il y a eu en Espagne plus de 700 morts entre 1975 et 1982 suite à la multiplication des actions protestataires, ce qui place l'Espagne à la hauteur de l'Italie durant les années de plomb. Il est donc impossible de parler d'une transition planifié et exemplaire, exempte de conflits et de problèmes puisque ces problèmes ont même failli venir à bout du processus démocratique lors du coup d'État du 23 février 1981, qui est un épisode encore largement tabou.

D'autres auteurs vont beaucoup plus loin. Les travaux de Bénédicte André-Bazzana (Mitos y mentiras de la Transición, El Viejo Topo, 2006) sont un véritable réquisitoire contre le mythe de la Transition. Il ressort de son étude que la Transition a été octroyée plus que négociée, que les forces démocratiques l'ont subie plus qu'accompagnée et qu'elles ont renoncé à beaucoup de leurs exigences pendant la phase décisive. Le consensus, plus apparent que réel, était le fruit de la nécessité, et non une collaboration volontaire, sans compter que pour l'imposer, les dirigeants en place, héritiers directs du franquisme, ont agité le spectre du coup d'État militaire et du conflit, une manière efficace de faire taire l'opposition démocratique et d'imposer la paix civile. C'est ainsi que l'opposition renonce à l'essentiel et impose ces renoncements à ses partisans et électeurs : acceptation de la monarchie et de ses symboles, acceptation de l'unité nationale - c'est-à-dire renoncement à une Espagne fédérale - et renoncement aux valeurs républicaines. Le consensus sert à occulter les déséquilibres des forces et à soutenir le thème de la réconciliation nationale nécessaire qui implique à son tour l'amnistie des crimes franquistes. Par ailleurs, la fausse image d'une transition partagée sert à cacher une entreprise de légitimation mutuelle : elle permet à l'opposition de masquer sa faiblesse et ses renoncements tout en valorisant sa participation, et elle donne aux forces héritières du franquisme une légitimité 
démocratique au travers $\mathrm{du}$ dialogue qu'elles disent conduire avec l'opposition démocratique. La démocratie permet à l'élite issue du franquisme de se maintenir au pouvoir et de conduire le processus de retour à la démocratie ainsi que d'accéder à l'ensemble européen, un des objectifs du processus. Mais pour les démocrates antifranquistes, ce retour à la démocratie a été une trahison: occultée, la mémoire démocratique anti-franquiste est réduite à être une simple mémoire démocratique, car valoriser l'anti-franquisme aurait ôté sa légitimité à l'élite au pouvoir, issue des rangs de la dictature (le roi en tout premier lieu) et qui conduisait le processus de passage à la démocratie - le danger était grand d'en faire des usurpateurs. On ne peut pas exclure qu'il y ait eu aussi une volonté des ex-franquistes de disqualifier la mémoire démocratique des vaincus, et de la République, d'où l'équation «mémoire de la résistance antifranquiste = danger pour le processus démocratique (réveil de conflits potentiels) », ce qui a conduit à l'autocensure de l'opposition démocratique et à la marginalisation des discours dissidents. Cela dit, les guides de la Transition n'hésitaient pas à agiter le spectre de la Guerre civile pour appeler à la modération et réduire le radicalisme, si bien qu'il fallait se rappeler la Guerre civile sans se rappeler pour quelles valeurs et contre quel ennemi on avait combattu. C'est ainsi que les forces démocratiques ont sacrifié leurs idéologies sur l'autel de la modération - et ont renoncé à tout ce qui leur donnait leur légitimité démocratique : leurs idéaux dans la lutte contre la dictature et le fascisme -, alors que les héritiers du franquisme n'avaient eux plus rien qui vaille à sauver (leur idéologie était épuisée) : seul comptait la nécessité de se sauver par le passage réussi à la démocratie. Ce processus a conduit à une exclusion du peuple comme acteur et à sa démobilisation volontaire au bénéfice d'accords secrets entre élites. Il fallait exclure toute polarisation politique et tout débat, privilégier la modération et la concorde, et bannir le radicalisme et le conflit. Autant de choses qui ne pouvaient qu'être nuisibles à la démocratie à terme. La mémoire de la Transition se construit donc en opposition à la mémoire de la Guerre civile et de la République. La gauche modérée est responsable de l'assassinat de la mémoire démocratique de la lutte anti-franquiste et de la disparition de tout un univers symbolique de références. En légitimant les héritiers du franquisme, elle ôte tout sens à la lutte anti-franquiste des démocrates. À cela il convient d'ajouter l'abandon de toute forme de radicalisme, d'utopie, en faveur d'une politique modeste, pragmatique, réaliste. C'en est fini du grand soir et des matins qui chantent: la démocratie devient une simple modalité de gestion de l'espace politique.

\section{Modèle ou échec ? Une critique radicale de la Transition espagnole du point de vue de la culture politique}

Le politologue Vicenç Navarro va encore plus loin en jugeant la Transition inmodélica sur ses résultats. Outre ses nombreux articles, comme par exemple celui publié par le quotidien El País, le8 janvier 2003, "Consecuencias de una transición inmodélica », il est l'auteur de l'ouvrage Bienestar insuficiente, democracia incompleta. Sobre lo que no se habla en nuestro país (Barcelone, Anagrama, 2002) dont le dernier chapitre, intitulé « Les racines de notre déficit démocratique », se décline en une série de sous-titres très évocateurs : « Un silence assourdissant ", " Réconciliation oui, oubli, non », "La transition incomplète ", «La transition n'est pas exemplaire ", "Insensibilité démocratique », «Les coûts de la perte de mémoire ». 
26 Dans son analyse, il revient sur les circonstances de la Transition espagnole, en soulignant le déséquilibre entre des forces conservatrices très dominantes dans le processus et une gauche démocratique très affaiblie après des décennies de répression, ce qui a conduit à avoir deux versions très répandues dans les milieux conservateurs du pays de ce que fut et de ce qu'a signifié le coup d'État militaire de 1936, la dictature qui en est issue et la transition vers la démocratie. La première interprétation conservatrice dit que le coup d'État et la dictature ont été nécessaires pour rétablir l'ordre et corriger la situation intolérable créée par la République. Cette version admet que la répression a été parfois excessive et que la dictature a été trop longue, mais maintient que le coup d'État militaire et la dictature restent quelque chose de positif pour l'Espagne. Cette position s'exprime dans la résistance aujourd'hui à condamner le franquisme, dans l'opposition à éliminer les symboles du régime (monuments au dictateur, aux figures du régime) et dans les hommages rendus aux intellectuels, aux hommes politiques ou religieux du régime. Selon cette version, la démocratie instaurée en Espagne par le roi nommé par Franco est le fruit de la volonté du régime d'ouvrir les institutions d'État à la gauche et aux nationalistes catalans et basques. Cette version est partagée par de nombreux membres du Parti populaire (droite). La seconde interprétation se définit elle-même comme centriste : elle assume que la Guerre civile fut un conflit entre les deux Espagnes (la «nationale» et la républicaine), conflit dans lequel les deux camps ont commis d'énormes atrocités - dues au caractère espagnol enclin à la violence - qu'il vaut mieux oublier. Elle défend la thèse du partage des responsabilités (équidistance) et affirme que la constitution de 1978 a mis fin à ces deux Espagnes. Parmi ceux qui défendent cette position, certains acceptent l'idée que les vaincus ont été maltraités et qu'ils ont droit à la reconnaissance et à des hommages comme cela a été le cas pour les vainqueurs. Cette équidistance doit aussi exister dans le domaine de la mémoire, et l'oubli imposé aux vaincus est une injustice. Ce point de vue est celui défendu par le journal El Pais, par exemple dans son éditorial du 8 août 2002.

Selon Vicenç Navarro, ces deux versions sont erronées. La réalité historique, rarement présentée dans les médias et dans les écoles, est différente. La République fut une des tentatives les plus profondes de moderniser les structures sociales archaïques de l'Espagne de l'époque. Elle a fait des réformes qui visaient des intérêts corporatistes (grands propriétaires terriens, groupes économiques et financiers, Banque, Église, Armée) et les classes sociales élevées, lesquelles ont répondu aux réformes par un coup d'État qui a stoppé net la modernisation et maintenu l'Espagne dans son retard économique, social, politique et culturel, portant préjudice à la grande majorité des Espagnols : en 1975 l'État providence espagnol était un des plus faibles de l'époque. Ce ne fut donc pas une moitié de l'Espagne contre une autre moitié mais une minorité contre une majorité de la population. D'où le besoin pour les forces putschistes de recourir à une grande répression et à la terreur, tant dans la zone républicaine que dans la zone nationale. La violence d'État, la politique de terreur de l'État franquiste ne peut être comparée aux débordements républicains que l'État républicain a toujours essayé de contrôler. Il serait donc juste de condamner le régime franquiste et d'interdire qu'on lui rende hommage. La vérité sur ce qui s'est passé en Espagne a donc été cachée par l'amnésie qui a accompagné l'amnistie et qui a porté préjudice à la culture démocratique espagnole.

Aujourd'hui, les nouvelles recherches historiques documentent la nature répressive du régime mais leur diffusion reste limitée car la culture populaire est très éloignée de la culture scientifique et elle est surtout très influencée par les médias télévisuels qui 
continuent à faire le silence sur la nature passée du régime avec lequel on a pactisé lors de la Transition.

Le coût politique de cette Transition, qui a imposé une révision de l'histoire récente, est une démocratie incomplète: le manque de volonté pour renouer avec la mémoire historique, de la République et de la dictature, a appauvri la démocratie espagnole, en limitant sa culture démocratique. Il a fallu par exemple attendre 2007 pour que l'État essaie de prendre en charge l'exhumation des corps jetés dans les fosses communes à la grande époque de la répression franquiste, ce qui est honteux pour la démocratie espagnole, condamnée récemment encore par l'agence des droits de l'homme des Nations unies.

La culture politique de l'Espagne actuelle ne peut donc pas s'appuyer sur une culture antifranquiste et démocratique bien établie, comme le prouve la permanence des symboles franquistes, conséquence logique d'une mémoire historique faible qui constitue aujourd'hui le principal déficit démocratique de la société espagnole. L'Espagne officielle peine à rendre hommage aux combattants anti-fascistes - pas de monuments aux morts ni de plaques pour les résistants - alors que l'armée espagnole prend en dépôt la mémoire franquiste, accueillant par exemple dans ses institutions les statues équestres de Franco enfin délogées des espaces publics, devenant ainsi la gardienne d'une mémoire antidémocratique. Et que dire de l'Église catholique espagnole: après avoir légitimé la dictature, elle est aujourd'hui encore incapable de demander pardon au peuple espagnol et, toujours encline à combattre la moindre tentative de laïcisation de l'État et de la société, elle est une source régulière de tensions sociales. Quant à la droite, elle peine à dénoncer un coup d'État militaire anti-démocratique et une dictature totalitaire, et tend sournoisement à réhabiliter la période dont elle est l'héritière (thèses révisionnistes de Pio Moa, Ricardo de la Cierva et Federico Jiménez Losantos, largement appuyés par les fondations du Parti populaire et par l'Église). Pour ce qui est de la gauche, après avoir fait le vide sur sa gauche, elle est devenue si modérée qu'elle en est à peine identifiable. Reste à examiner le cas de la Constitution : incapable de reconnaître et de prendre en compte le caractère plurinational de l'État, elle conduit aujourd'hui à une interrogation majeure sur le modèle d'articulation territoriale et à une crise de l'identité espagnole, attaquée de toutes parts par les nationalismes périphériques. La monarchie elle-même semble contestée, non seulement dans les stades mais plus sérieusement dans les mouvements civiques de protestation parsemés de drapeaux républicains.

31 Certes, les circonstances ont pu imposer les voies qu'a empruntées le passage à la démocratie en Espagne, mais aujourd'hui ce pays semble confronté aux limites de ce qu'il a érigé en modèle de transition pour le monde.

\title{
BIBLIOGRAPHIE
}

\author{
Bibliographie à la base de l'article
}

ANDRÉ-BAZZANA Benedicte, Mitos y mentiras de la transición, Madrid, El Viejo Topo, 2006. 
BABY Sophie, Violencia y transiciones políticas a finales del siglo XX. Europa del Sur-América latina, Madrid, Collection de la Casa Velázquez, 2009.

CUESTA BUSTILlo Josefina, La Odisea de la memoria, Historia de la memoria en España Siglo XX, Madrid, Alianza Editorial, 2008.

MATEOS Abdon, La España del presente: de la dictadura a la democracia, Madrid, Asociación de historiadores del presente, 2006.

MOLINERo Carme, La Transición, treinta años después, Barcelone, Península, 2006.

NAVARRo Vicenç, Bienestar insuficiente, democracia incompleta. Sobre lo que no se habla en nuestro país, Barcelone, Anagrama, 2002.

QUIROSA CHEYROUZE Y MUÑoz Rafael (coord.), Historia de la transición en España. Los inicios del proceso democratizador, Madrid, Biblioteca Nueva, 2007.

REDERO SAN ROMÁNManuel, Transición a la democracia y poder político en la España postfranquista (1975-1978), Salamanca, 1993.

-, La transición a la democracia en España, Madrid, Marcial Pons, 1994.

TUSELL Javier, La Transición española a la democracia, Madrid, Historia 16, 1991-1999.

—, Historia de la Transición, 1975-1986, Madrid, Alianza Ed, 1996.

VV. AA., Memoria de la Transición, Madrid, Taurus, 1996.

\section{Autres références}

CARR Raymond et FUSI Juan Pablo, España, de la dictadura a la democracia, Barcelone, Planeta, 1979. CORTES CABANILLAS Juan, Crónica de Juan Carlos Rey. De la Restauración a la Constitución, Alce, 1978. MARTín VILLA Rodolfo, Al servicio del Estado, Barcelone, Planeta,1984. MORÁN Gregorio, El precio de la Transición, Barcelone, Planeta, 1991.

PALACIO ATARD Vicente, Juan Carlos I y el advenimiento de la democracia en España, Madrid, EspasaCalpe, 1989.

POWELL Ch. T., El piloto del cambio. El rey, la monarquía y la Transición a la democracia, Barcelone, Planeta, 1991.

PREGO Victoria, La Transición (los años más decisivos de nuestra historia reciente), 1993.

PRESTON Paul, El triunfo de la democracia en España (1969-1982), Barcelone, Plaza y Janés, 1986.

\section{RÉSUMÉS}

Dans le vaste champ d'étude que constituent les phénomènes de transition et de consolidation des démocraties, l'Espagne reste une référence incontournable. Très vite présentée comme exemplaire par l'historiographie dominante, la Transition espagnole devient à la fois un modèle pour de nombreux pays d'Amérique latine et d'Europe de l'Est et le nouveau mythe fondateur de la jeune démocratie espagnole - sous l'impulsion des partis politiques et des médias qui la célèbrent. Un mythe fortement remis en question aujourd'hui par la nouvelle production historiographique espagnole et française, mais aussi par la réalité de la culture politique du pays elle-même. 
For all those who study democracies' transitional and consolidating phenomena, Spain remains a key reference. Hailed almost from the start as unique by the dominant historiography, the Spanish Transition became both a model for many South American and Eastern European countries, and the founding myth for the newly-born Spanish democracy-a phenomenon made possible through the impulse of the political parties and the media that celebrated it. This myth is now questioned by the new Spanish and French historiography, as well as by the country's actual political culture.

\section{INDEX}

Keywords : 20th-21st-century Spanish Historiography, 20th-21st-century Spanish History, 20th21st-century Spanish Political Culture, Conflicts, Democracy, Historical Memory, Political Myth, Spain, The Spanish Transition

Mots-clés : conflits, culture politique espagnole 20e-21e siècles, démocratie, Espagne, Histoire Espagne 20e-21e siècles, Historiographie Espagne 20e-21e siècles, mémoire historique, mythe politique, Transition espagnole

\section{AUTEUR}

\section{CHRISTIAN DEMANGE}

Maître de conférences à l'université Stendhal-Grenoble 3, Centre d'études et de recherches hispaniques (CERHIUS) au sein de l'Institut des langues et des cultures d'Europe et d'Amérique (ILCEA) 